\title{
In-Chamber Reel-to-Reel System for Random-Access Volume Electron Microscopy
}

\author{
S. Mikula ${ }^{1}$ \\ 1. Electrons - Photons - Neurons, Max-Planck Institute of Neurobiology, Martinsried, Germany.
}

Brain-wide synaptic wiring diagrams will be necessary to accurately predict brain-wide circuit computations underlying the diverse behaviors produced by an organism. Currently only volume electron microscopy (VEM) provides the nanometer-scale resolution required to reliably and precisely reconstruct arbitrary neuronal circuits contained within the imaged volume. Given that VEM datasets for large volumes may exceed petabytes, the automation of high-throughput VEM is critical for scaling up to mammalian whole-brains, such as the mouse or Etruscan pygmy shrew [1].

One approach for automating VEM involves automated tape-collecting ultramicrotomy (ATUM), essentially a motorised conveyor belt for picking up serial ultrathin sections that are produced by conventional ultramicrotomy [2]. However, ATUM does not completely automate VEM as the sections collected on tape must be cut into strips and adhered to silicon wafers for subsequent scanning electron microscopy (SEM). A more convenient route would be to directly image the sections-on-tape without further manual modifications, such as would be provided by an in-chamber reel-to-reel system.

In-chamber reel-to-reel systems have been described for transmission electron microscopy, though they have not been implemented, nor shown compatible with ATUM [3,4]. Here I show the design and implementation of a compact in-chamber reel-to-reel system for SEM that is compatible with sections collected on tape via ATUM, allowing for automation of the complete serial-section VEM workflow. Full automation through software is currently in progress.

The design of the in-chamber reel-to-reel system consists of source and target reels actuated by DC rotary micromotors (Faulhaber, Schönaich, Germany) to advance the tape forward or backward (Figure 1a-c) using a programmable power supply. The tape is advanced with tension across a flat conductive surface, allowing it to remain flat for imaging, which minimises autofocus corrections during mosaic acquisitions since a planar approximation for working distance may be used. Hi-vac imaging requires conductive tape and connecting the flat conductive surface to ground.

An implementation of the design was tested on an FEI Quanta 200 SEM with back-scattered electron (BSE) imaging in hi-vac (Figure 1d-e). Serial $100 \mathrm{~nm}$-thick mouse whole-brain sections were generated from a BROPA-like sample [5] and collected onto $20 \mu \mathrm{m}$-thick copper tape using an ATUMtome (RMC Boeckeler, Tucson, USA). High-resolution SEM confirmed the absence of sample charging and motorinduced vibrations and electronic noise (Figure 1f). Advancing the tape to image adjacent sections was possible but imprecise, though it is expected that motor encoders will improve targeting tape positions.

The compact design of the in-chamber reel-to-reel system makes it compatible with both FEI and ZEISS SEMs and allows for direct placement onto the SEM XYZ stage, enabling mosaic acquisitions of sections-on-tape without additional motors. Combined with ATUM, the in-chamber reel-to-reel system offers a solution for fully automated VEM, which will be necessary for imaging mammalian wholebrains. In addition, the ability of the in-chamber reel-to-reel system to randomly-access any part of the sections-on-tape allows for random-access VEM, a more efficient route than bulk imaging to reconstruct 
brain-wide neuronal circuits. The promise of reconstructing arbitrary brain-wide circuits, even on conventional single-beam SEMs, will make the in-chamber reel-to-reel system an essential technology for brain-wide cellular connectomics [6].

\section{References:}

[1] S. Mikula. Progress Towards Mammalian Whole-Brain Cellular Connectomics. Frontiers in Neuroanatomy, 62. (2016)

[2] R. Schalek et al. Development of High-Throughput, High-Resolution 3D Reconstruction of LargeVolume Biological Tissue Using Automated Tape Collection Ultramicrotomy and Scanning Electron Microscopy. Microscopy and Microanalysis, 17(Supplement S2), 966-967 (2011)

[3] K. Hayworth \& A. Hayworth. Methods and Apparatuses for the Automated Production, Collection, Handling, and Imaging of Large Numbers of Serial Tissue Sections.

http://www.google.com/patents/US20060008790. (2006)

[4] C.S. Own et al. Reel-to-Reel Electron Microscopy: Latency-Free Continuous Imaging of Large Sample Volumes. Microscopy and Microanalysis, 21(S3), 157-158. (2015)

[5] S. Mikula \& W. Denk. High-Resolution Whole-Brain Staining for Electron Microscopic Circuit Reconstruction. Nature Methods, 12(6), 541-546. (2015)

[6] The author acknowledges funding from the Max Planck Society and thanks Winfried Denk for his support, Pat Brey, Ken Hayworth and Yoshiyuki Kubota for many useful discussions, Juergen Tritthardt for electronics support and Debbie Zapoglou for CAD support.
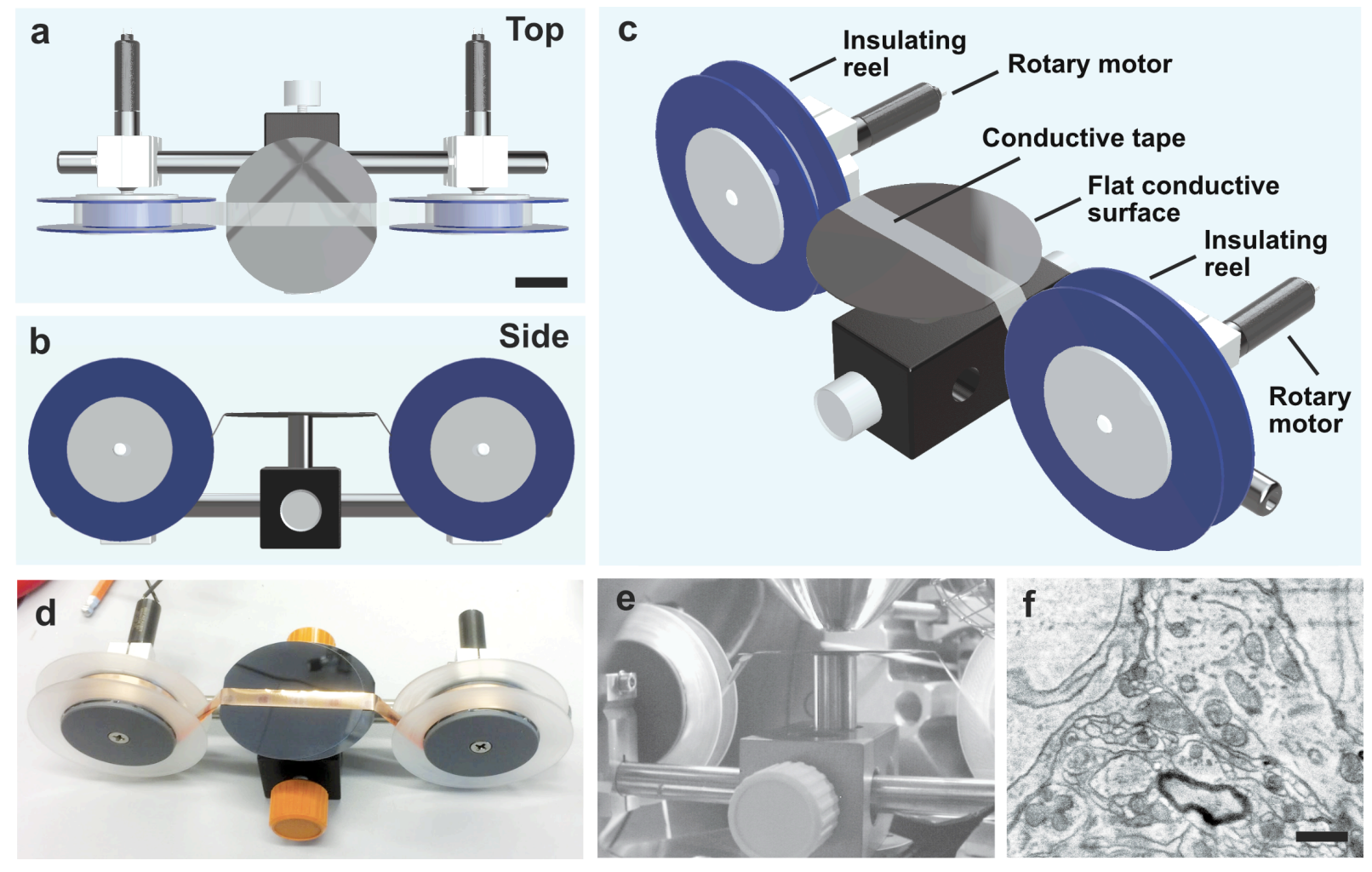

Figure 1. (a-c) CAD diagrams for the in-chamber reel-to-reel system. (d) Implementation of the reel-toreel system. (e) Chamber-scope image of the reel-to-reel system inside an FEI Quanta 200 SEM. (f) High-resolution SEM of whole-brain sections-on-tape imaged at hi-vac in an FEI Quanta 200 SEM using a custom BSE detector. Scale bar in a is $2 \mathrm{~cm}$ and $\mathrm{f}$ is $1 \mu \mathrm{m}$. 